\title{
Interference Model and Evaluation in LTE Networks
}

\author{
Nourredine Tabia*, Alexandre Gondran+, Oumaya Baala*, Alexandre Caminada* \\ * UTBM, SET \\ Belfort, France \\ + ENAC, \\ Toulouse, France \\ \{nourredine.tabia, oumaya.baala, alexandre.caminada\}@utbm.fr, alexandre.gondran@enac.fr
}

\begin{abstract}
The purpose of this paper is to evaluate the impact of frequency parameter settings while evaluating interference model in LTE (Long Term Evolution); the work is applied on a network. We develop and validate the interference model based on SINR which is used by 3GPP (3rd Group Partnership Project) to estimate the quality of signal received by $U E$ (User Equipment) and issued from eNB (enhanced Node Base). For this aim, two frequency schemes have been compared: the frequency reuse 1 scheme whereby the whole available bandwidth is used in each cell/sector and the frequency reuse 3 scheme in which the entire bandwidth is divided into 3 non-overlapping groups and assigned to 3 co-site sectors within each cell.
\end{abstract} factor

Keywords-LTE; SINR;Interference; frequency reuse; load

\section{INTRODUCTION}

Multiple access schemes in LTE system network have attracted an increasing interest in the last years [9]. The main performance targets are to improve the spectral efficiency 2 to 4 times compared to HSDPA Release 6 in which peak rates can reach $100 \mathrm{Mbps}$ on downlink using Orthogonal Frequency Division Multiple Access (OFDMA) and 50Mbps on uplink using Single Carrier Frequency Division Multiple Access (SC-FDMA). These techniques provide orthogonality between the users, reduce interference and improve the network's Quality of Service (QoS). In addition, to make the network design process time-efficient, Self-Organizing Network (SON) functionalities added within LTE architecture by incorporating automated optimization, can significantly reduce deployment and maintenance costs (CAPEX and OPEX for CAPital EXpenditure and OPerating EXpenditure respectively).

In a wireless network with several cells, the main key to improve the achievable average throughput by user is to mitigate the inter-cell interference caused by using the same frequency within adjacent cells. Various frequency reuse schemes have been proposed in literature $[1,2]$. Mitigation technique is generally categorized into three major techniques as shown in [3]: interference cancellation, interference averaging and interference avoidance techniques. The focus in this paper is on avoidance through inter-cell coordination, to achieve better network performance goals.
Usually, all the schemes refer to a fractional frequency reuse technique. Two known variants of fractional frequency reuse can be found in literature. Firstly, the Partial Frequency Reuse which has been adopted in the 3GPP LTE systems for the first time in [4]. This technique improves considerably the interferences at cell edge but loses capacity due to the partitioning of available spectrum [2]. The main idea is to partition the cell into two static zones; the central area of the cell uses generally $2 / 3$ of the available spectrum while the edge area of the cell uses a third of the $1 / 3$ reserved for the co-site three adjacent cells. A detailed description of Partial Frequency Reuse can be found on [3]. Secondly, the Soft Frequency Reuse is another variant of fractional frequency reuse presented in [5] and [1]. This scheme imposes some restrictions in terms of allocation of frequency and power in the cell-center and cell-edge areas. The advantage of this reuse pattern is that it provides enhanced services to the UE located at the boundary of the cell. This technique allows the system to use higher power in the boundary than in the center of the cell and the frequency dynamically allocated to the edge of the cell must be orthogonal to the adjacent cells to mitigate the inter-cell interference. Both techniques, Partial and Soft Frequency Reuse, are generally known as Inter-Cell Interference Coordination (ICIC); they require the planner to develop coordination function between eNB.

Resource allocation in radio networks essentially depends on the quality of the some reference signals received by the UE. In LTE, they are the Reference Signal Received Power (RSRP) and the Reference Signal Received Quality (RSRQ) as code power on Common Pilot (RSCP) and Ec/No in UMTS. Each user is assigned a portion of the spectrum depending on RSRP and RSRQ. Then the more complex optimization of reference signal is the RSRQ one which is based on Signal to Interference plus Noise Ratio (SINR) [2, 6]. SINR is an important performance indicator for estimating the achievable throughput, taking into account the interference received from the neighboring cluster of first-tier cells. The estimation and optimization of the SINR are well-known problems in radio communication systems such as 802.11, GSM or UMTS [11, 12, 13], and LTE needs also a good estimation and control of SINR. 
In LTE, several parameters investigations have been developed to meet requirement set. Various combinations of antenna parameter settings have been studied in terms of SINR and throughput performance outlined for example in $[6,10]$. We propose in this paper to evaluate both SINR and throughput models for different frequency assignment schemes for LTE on the basis of a real UMTS network for which the traffic load is transferred on LTE mode. The work allows us to get a thin analysis of UE on and off links during a full day of traffic load with a lot of variations along time. The remainder of this paper is structured as follows. Section II introduces the system model and the basic assumptions. Section III extends this work and shows the performance metrics and the test assumptions. Section IV presents some results to highlight the use of SINR interference model for both reuse 1 and 3 . Conclusions and perspectives are drawn in section $\mathrm{V}$.

\section{SYSTEM MODEL}

\section{A. Basic Assumptions}

In this paper we consider the downlink transmission from eNB to UE and at first we illustrate the interference schemes using a theoretical model of seven-cell hexagonal layout as shown in Figure 1. Three sectors are considered in each site with three base stations. In (a) we see the frequency reuse $1 \times 3 \times 1$ pattern where 1 site with 3 sectors use 1 frequency set, that is the same frequency set for all sectors. In (b) we see the frequency reuse $1 \times 3 \times 3$ pattern where 1 site with 3 sectors use 3 frequency sets, that is different frequency set for all sectors. The $1 \times 3 \times 3$ requires a frequency plan but should avoid interference when the network load becomes too high to apply the $1 \times 3 \times 1$ pattern.

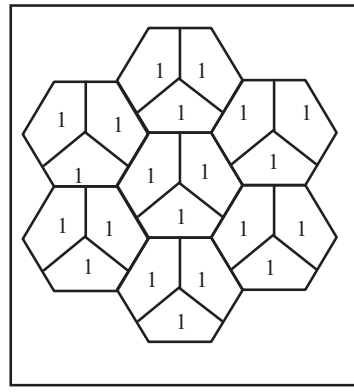

a)

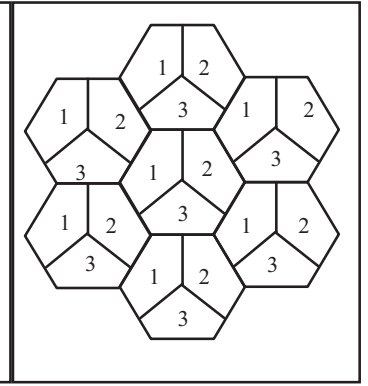

b)
Figure1. Seven-cell hexagonal layout. a) Reuse 1 b) Reuse 3

The features of our computational model are the following:

1) Intra-frequency interference is avoided due to the use of OFDMA technique in downlink transmission. In LTE the orthogonality between subcarriers insures that the interference inside the cell can be ignored.

2) The basic resource element in OFDMA is the physical resource block (PRB) which spans both frequency and time dimensions. In this paper, we do not take into account PRB to estimate the inter-cell interference; we only focus on the frequency sub-band reuse scheme. Two adjacent cells are scrambling each other if they are using the same sub-band to transmit data as shown in Figure 2. It gives a worst but fast estimation of SINR.
3) Scenarios are used to represent the traffic distribution of our model since LTE network traffic is unpredictable now. The interference model constructed here and based on SINR depends not only on antenna parameters (frequency, tilt, azimuth...) but also on traffic load along day; then in our computation the users available in target sector or adjacent sectors can affect the received signal from other users. As we consider that all users communicate at the same time, a scenario shows the distribution of demand at a given time.

4) We introduce a performance indicator to measure occupancy rate on each sector, the load factor. This indicator is a ratio between used bandwidth and the maximum available bandwidth. It is measured for each sector of the network. The feature of the load factor is to determine the bottlenecks of the network and the overloaded sectors. This indicator will be used in the interference model.
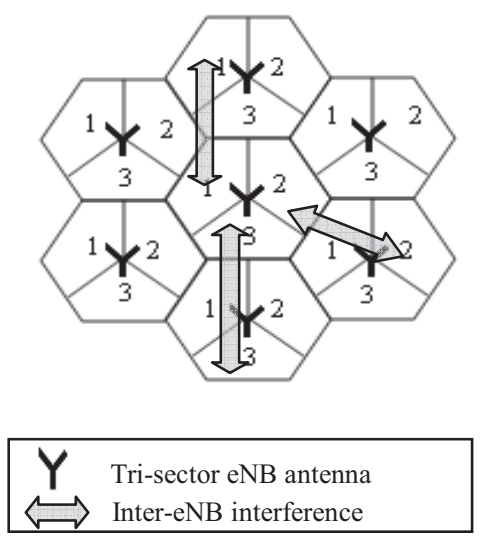

Figure2. Inter-cell interference

\section{B. Case Study and Problem Formulation}

The considered network for this study consists of trisectors sites in the city of Belfort, located in the northeastern of France. Service area is shown Figure 3; it is a $40 \mathrm{~km} \times 20 \mathrm{~km}$ area with a lot of big industrial companies and the population is about 200,000 people, which includes residents and people coming from outside the area for work. For our model the service area is divided into a grid of equally sized bins. A bin is a $25 \times 25$ meters area.

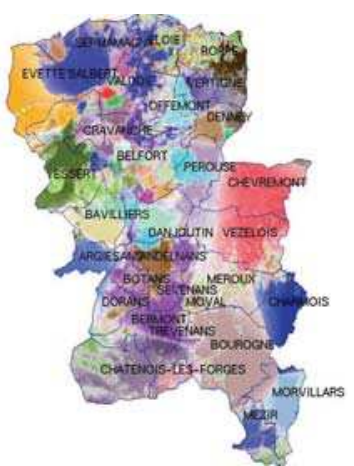

Figure3. Coverage per transmitter for the baseline network [City of Belfort] 
Due to the very small size of the bin, we assume the same signal propagation conditions within a bin; it means that all users located inside a bin have the same signal and same RSRP, SINR and RSRQ. Thus, a bin determines the resolution of the computation and the amount of data on signals. A bin is characterized by its number of users and the category of required services for each user (voice, data...). Each sector in the network is equipped with one directive antenna. Each antenna is characterized by its parameters: radiation pattern, azimuth, electrical and mechanical tilt, gain in transmission and reception, frequency of transmission and output power in downlink. Due to the dynamic aspect of the network and changes in traffic demand, we introduce the concept of traffic scenarios. A scenario is a given distribution and load of the traffic demand at a given time for each bin of the map. Then several scenarios allow us to compute different situation of network performance.

The problem formulation is given by the following sets of data, parameters and functions.

Let: $B=\left\{1, \ldots, n^{B}\right\}$, the set of $n^{B}$ base stations eNB of the network; $T=\left\{1, \ldots, n^{T P}\right\}$, the set of bins of the map; and $C_{t, s}$, the number of users located on the bin $t$ in scenario $S$.

The interference model based on SINR is thus calculated as defined in Equation 1:

$$
\text { II. } \gamma_{b, t, s}=\frac{p_{b, t}^{R} f_{b}}{\sum_{b^{\prime} \neq b, f_{b}=f_{b^{\prime}}} p_{b^{\prime}, t}^{R} f_{b^{\prime}} \delta_{b, s} \delta_{b^{\prime}, s}+n_{0} w}
$$

Where, $\gamma_{b, t, s}$ is the SINR received by the bin $t$ and issued from the eNB $b$ in scenario $s ; f_{b}$ and $f_{b^{\prime}}$ are the frequencies used by eNB $b, b^{\prime}$ respectively; $\delta_{b, s}$ and $\delta_{b^{\prime}, s}$ are load factors that corresponds to base stations $b$ and $b^{\prime}$ in scenario $s$. Base station $b$ is said to be saturated in scenario $S$ if its load factor $\delta_{b, s}$ is equal to 1. The term $w$ represents the total bandwidth used by $b$ and $n_{0}$ is the thermal noise over the bandwidth $w$.

The terms $p_{b, t}^{R}$ and $p_{b^{\prime}, t}^{R}$ are the end power received by UE located in bin $t$ from respectively $b$ and $b$ '. The estimation of this power is given by the Hata propagation model in Equation 2:

$$
\begin{aligned}
p_{b, t}^{R}(d B m)= & p_{b}^{E}-P L_{b, t}+g_{b}^{M A X}-a_{b}^{V E R}\left(\theta_{b, t}-t_{b}^{M}-t_{b}^{E}\right)- \\
& a_{b}^{H O R}\left(\varphi_{b, t}-a_{b}\right)
\end{aligned}
$$

Where, $p_{b}^{E}$ is the power in $\mathrm{dBm}$ issued from the eNB $b \cdot g_{b}^{M A X}$ is the antenna gain while $a_{b}^{V E R}$ and $a_{b}^{H O R}$ are the vertical and horizontal radiation pattern due to the position of the bin from the main beam of the antenna. As shown in Figure 4(a), $a_{b}^{V E R}$ depends essentially on the antenna tilt which is the angle of the main beam below the horizontal plane. We distinguish two different tilts: the mechanical tilt $t_{b}^{M}$ to adjust the physical angle of the antenna brackets and the electrical tilt $t_{b}^{E}$ which does not change the physical angle, but adjusts the radiating currents in the antenna elements to lower the beam in all horizontal directions.

The right part of Figure 4 shows the impact of azimuth parameter on the horizontal radiation pattern. The azimuth is the horizontal angle $a_{b}$ between the north and the antenna main lobe direction.

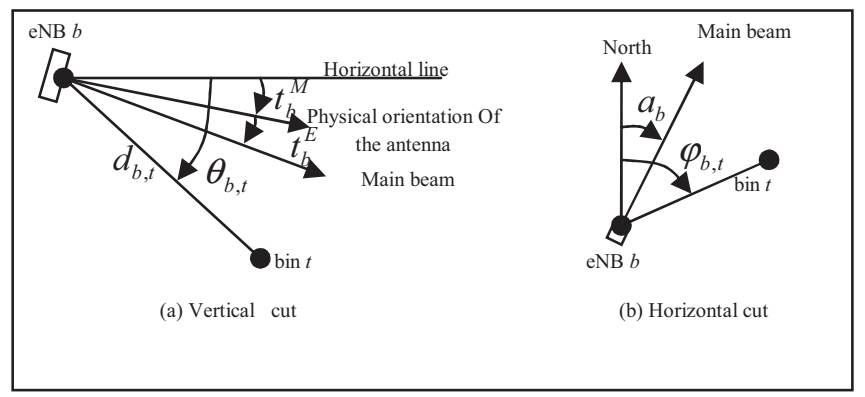

Figure4. Horizontal and vertical angles

In 3GPP LTE tests, we apply the two formulas [6] given by Equation 3 and Equation 4 for the computation of $a_{b}^{V E R}\left(\theta_{b, t}, t_{b}^{M}, t_{b}^{E}\right)$ and $a_{b}^{H O R}\left(\varphi_{b, t}, a_{b}\right)$ :

$a_{b}^{V E R}\left(\theta_{b, t}, t_{b}^{M}, t_{b}^{E}\right)=-\min \left[12\left(\frac{\theta_{b, t}-t_{b}^{M}-t_{b}^{E}}{\theta_{3 d B}}\right), S L A_{v}\right], S L A_{v}=20 d B$

$a_{b}^{H O R}\left(\varphi_{b, t}, a_{b}\right)=-\min \left[12\left(\frac{\varphi_{b, t}-a_{b}}{\varphi_{3 d B}}\right), A_{m}\right], A_{m}=25 d B$

Where $S L A_{v}$ is the side lobe attenuation and $A_{m}$ is the front-to-back attenuation [10]. $\theta_{3 d B}$ and $\varphi_{3 d B}$ are the half power beam width in vertical and horizontal plan respectively.

Finally, with the path loss the Hata model for urban areas is formulated as in Equation 5:

$$
\begin{aligned}
P L_{b, t}= & 69.55+26.16 \log \left(f_{0}\right)-13.82 \log \left(z_{b}\right) \\
& -a\left(z_{t}\right)+\left(44.9-6.55 \log \left(z_{t}\right)\right) \log \left(d_{b, t}\right)
\end{aligned}
$$

And for small or medium sized city the value of $a$ is:

$$
a\left(z_{t}\right)=0.8+\left(1.1 \times \log \left(f_{0}\right)-0.7\right) z_{t}-1.56 \log \left(f_{0}\right)
$$

Where, $P L_{b, t}$ is the path loss $(\mathrm{dB})$ in urban area between the eNB $b$ and the bin $t, f_{0}$ is the frequency of transmission (Mhz), $z_{b}$ and $z_{t}$ are the height of the base station $b$ and the bin $t(\mathrm{~m}), d_{b, t}$ is the distance between the base station $b$ and the center of the bin $t(\mathrm{~m})$, while $a\left(z_{t}\right)$ is the correction factor for mobile unit antenna height $(\mathrm{dB})$.

\section{Test Assumptions ANd Performance Metrics}

We aim at evaluating the SINR model to identify where frequency reuse presents a remarkable increase of covered users with respect to antennas parameters settings. The main parameters and assumptions we used 
are those selected by 3GPP for LTE as shown in Table 1. Evaluations are performed by a static snapshot of the network level.

\begin{tabular}{|c|c|}
\hline Parameters & Simulation setting \\
\hline Network layout & 35 sites and 88 sectors \\
\hline System frequency & $1800 \mathrm{Mhz}$ \\
\hline System bandwidth & $20 \mathrm{Mhz}$ \\
\hline Frequency reuse factor & 1 and 3 \\
\hline eNB heights range & {$[17 \mathrm{~m}, 46 \mathrm{~m}]$} \\
\hline UE height & $1.5 \mathrm{~m}$ \\
\hline Propagation loss model & Hata model \\
\hline TX power range & [39 dBm, $46 \mathrm{dBm}]$ \\
\hline Mechanical tilt range & {$\left[0^{\circ},-6^{\circ}\right]$} \\
\hline Electrical tilt range & {$\left[0^{\circ},-10^{\circ}\right]$} \\
\hline Azimuth range & {$\left[0^{\circ}, 360^{\circ}\right]$} \\
\hline Horizontal HPBW & $+70^{\circ}$ \\
\hline Vertical HPBW & $+10^{\circ}$ \\
\hline Antenna gain range & {$[14 \mathrm{dBi}, 18.9 \mathrm{dBi}]$} \\
\hline Traffic distribution & $\begin{array}{l}\text { Distribution in } \\
\text { proportion to UMTS } \\
\text { traffic load }\end{array}$ \\
\hline
\end{tabular}

Table1. Test assumptions for LTE downlink

Various performance metrics are considered herein:

\section{A) Signal to Interference plus Noise Ratio}

The SINR, expressed in Equation 1, is an important indicator to evaluate cellular networks. SINR choice is motivated by the fact that:

- It takes into account all the parameters of the antenna.

- It depends on the traffic distribution and the load factor of the network.

- It resizes the network and determines which base station controls each user.

- It allows us to calculate the total throughput of the network.

\section{B) Load Factor}

The Load Factor of the sector/cell is the ratio between the total allocated bandwidth for the cell which is the required bandwidth and the maximum total bandwidth available in the cell which is the resources allocated to the cell. Let $\delta_{b, s}$ the load factor, then: $\delta_{b, s}=\frac{w_{b, s}^{s}}{w}$. Where, $w_{b, s}^{S}$ in the total allocated bandwidth to the base station $b$ in the reference scenario $s$, and $w$ is its maximum available bandwidth. It is worthwhile to mention that load factor is one of the main key indicators. It has been suggested that the downlink cell load for a stable network should not exceed 70\% [7]. Huge loaded cells are those for which $\delta_{b, s}>0.7$ and overloaded cells are those for which $\delta_{b, s}>1$.

\section{C) Throughput}

We used the SINR to determine the throughput offered by a base station to the set of users who are located in the cell bins: the higher the SINR, the greater the quality of the channel and the throughput. The Table 2 below gives the current correspondences between SINR, throughput and modulation [9]. The user is in outage if its SINR is below the required threshold for the most robust MSC.

\begin{tabular}{|c|c|c|c|}
\hline Index & $\begin{array}{c}\text { Modulation and } \\
\text { coding }\end{array}$ & $\begin{array}{c}\text { Throughput } \\
{[\mathrm{Bits} / \mathrm{s} / \mathrm{Hz}]}\end{array}$ & $\begin{array}{c}\text { SINR } \\
{[\mathrm{dB}]}\end{array}$ \\
\hline 0 & Outage & 0 & $<0.9$ \\
\hline 1 & QPSK 1/3 & 0.75 & 0.9 \\
\hline 2 & QPSK 1/2 & 1 & 2.1 \\
\hline 3 & QPSK 2/3 & 1.25 & 3.8 \\
\hline 4 & 16QAM 1/2 & 2 & 7.7 \\
\hline 5 & 16QAM 2/3 & 2.75 & 9.8 \\
\hline 6 & 16QAM 5/6 & 3.25 & 12.6 \\
\hline 7 & 64QAM 2/3 & 4 & 15.0 \\
\hline 8 & 64QAM 5/6 & 5 & 18.2 \\
\hline
\end{tabular}

Table2. Modulation and coding of required SINR values and associated throughput [Schoenen08]

\section{RESUlTS}

In order to evaluate the SINR model presented in section II, we focus on frequency reuse 1 ( $1 \times 3 \times 1$ pattern) and 3 (1x3x3 pattern). The baseline network used for our study is the city of Belfort described in section II.2. The UE are randomly dropped in each cell in proportion to UMTS traffic load. We present in the following the methodology to evaluate the SINR model taking into account traffic data of the baseline network.

First of all, we assume that the base station is engaged to communicate with a UE if the SINR received by the UE is higher enough, i.e. achieve the required SINR threshold, to establish a communication. So, the UE is allocated to a base station according to the quality of the received SINR. We further assume that the load factor is considered here to calculate the SINR as mentioned in Equation 1.

A cell is defined as a set of bins of the map; a bin is assigned to the base station which presents the best SINR. As a first step we assign the bin to the base station on the basis of the best RSRP. This is defining the initial cell coverage for each station. Then from this we know the number of UE per cell and the traffic demand for the 
station. We compute the load factor for each station, and finally we do the computation of the SINR which is linked to the RSRQ. As a second step we assign again the bin to the base station on the basis of the best SINR. From that we estimate again the load factor for each station. This second step is repeated several times until we get a stable network configuration

The traffic data we used comes from one real UMTS network. The tests consider three different scenarios originating from the traffic of one day, as shown in Figure 5. Three scenarios were selected at different times of the day as follows: a first scenario at 8am with low traffic and 482 users dropped randomly in the network; a second scenario at $3 \mathrm{pm}$ with average traffic and 1,019 users; and a third scenario at $6 \mathrm{pm}$ with high traffic and 1,471 users. We are considering that all users are accessing the network at the same time (saturated traffic condition).

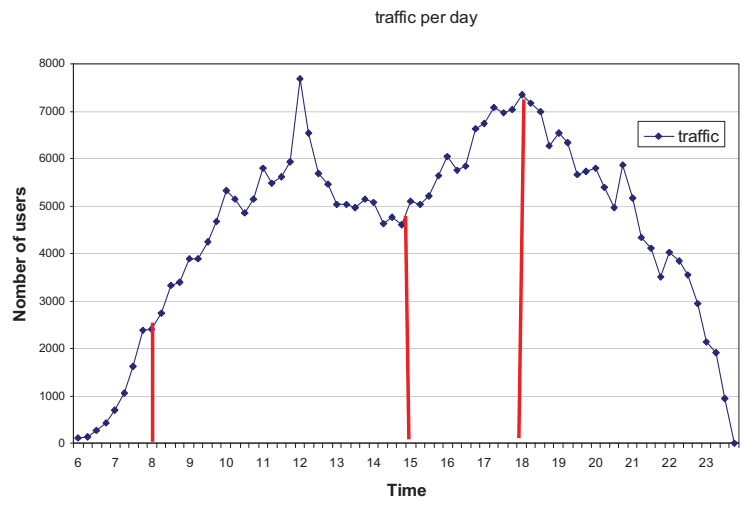

Figure5. Example of day traftic with three chosen scenarios

The Figure 6 shows the concentration of the traffic in the $3^{\text {rd }}$ scenario at $6 \mathrm{pm}$. The traffic is represented by a color gradient. The light color shows a low traffic, while the dark color shows higher traffic.

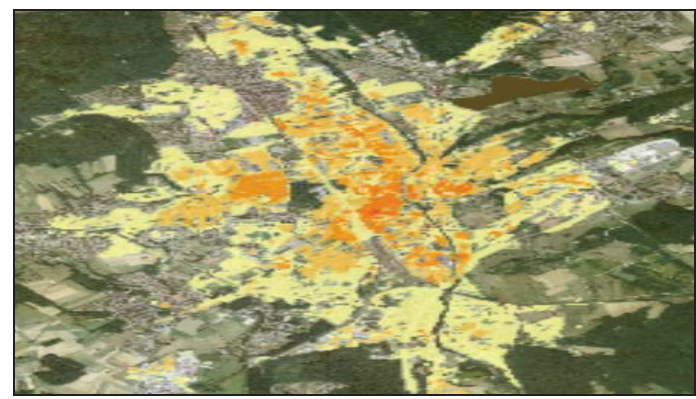

Figure6. Example of traffic concentration at $6 \mathrm{pm}$

To compare the performances of both reuse 1 and reuse 3 patterns, the number of users in outage is used as an important metric. The minimum required SINR is, as shown in Table 2, equal to $0.9 \mathrm{~dB}$. Below this value of SINR, one user cannot establish a communication as defined in Equation 6:

$$
\gamma_{b, t, s}=\frac{p_{b, t}^{R} f_{b}}{\sum_{b^{\prime} \neq b, f_{b}=f_{b^{\prime}}} p_{b^{\prime}, t}^{R} f_{b^{\prime}} \delta_{b, s} \delta_{b^{\prime}, s}+n_{0} w}<\gamma^{M I N}
$$

Figures 7 and 8 depict the number of users in outage obtained with both reuses pattern: scheme 1 whereby the whole available bandwidth is used in each sector, and scheme 3 whereby the entire bandwidth is divided into 3 non-overlapping parts and assigned in three neighbouring sectors within one site. In the network design we test for scheme 3 , the sub-band assignment is done in function of azimuth orientation of the sectors. Sectors in opposite direction from neighbor sites where assigned different frequency groups.

The program implementing our model is developed in $\mathrm{C}++$. We run the program about ten times to get stable results; two examples are presented in the following to show the interest of using the reuse scheme 3 in a real network design and traffic load from the computation of the SINR.

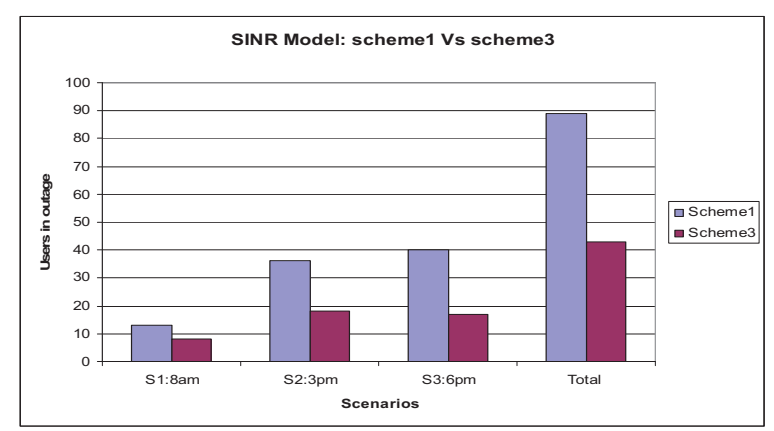

Figure7. Reuse scheme 3 within 3 scenarios

In Figure 7 we see that the number of users in outage in LTE is more important in scheme $1(13,36$, and 40) than in scheme $3(8,18$ and 17) for the three scenarios of traffic load considered here $(8 \mathrm{am}, 3 \mathrm{pm}$ and $6 \mathrm{pm}$ respectively). In Figure 8 we did another test with two other traffic conditions at 12 o'clock and $10 \mathrm{pm}$, it shows results in the same direction. These results are consistent since the total bandwidth used in pattern 1 can assign more resources, but would jeopardize the rate offered by the network. This is due mainly to the undesirable intercell interference generated by neighboring cells using the same frequency set. Such a dense reuse is an obvious pitfall which limits the throughput at the cell edge. We can also note according to these results that, the higher the traffic, the higher the number of outage users, whatever the frequency pattern solution.

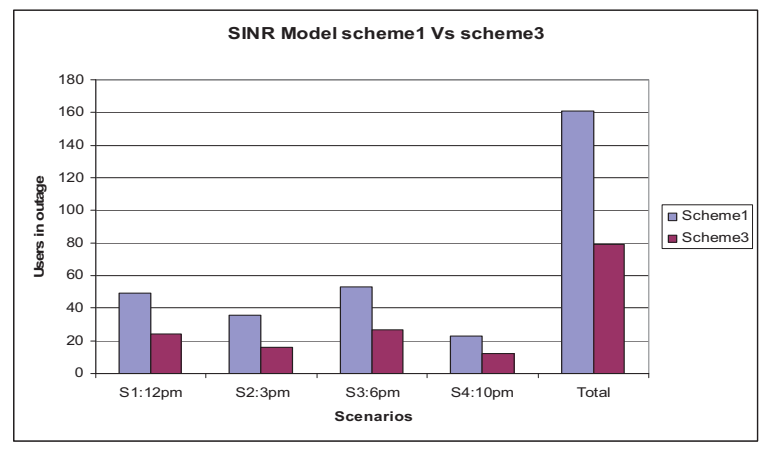

Figure8. Reuse scheme 3 within 4 scenarios

Where $\gamma^{M I N}=0.9 d B$ 


\section{CONCLUSION}

In this paper we present the interference modeling and evaluation in LTE network. The analysis has been carried out using two reuse schemes of frequency called $1 \times 3 \times 1$ and $1 \times 3 \times 3$ and the load factor which represents the traffic density of each cell of the network. Our computation uses the Hata propagation model to measure the radio signal propagation in a medium sized city in France from one UMTS network. We examine how the interference model behaves under realistic traffic scenarios, so we have considered different time of traffic load to represent the traffic growth and demand during the day; three scenarios were selected with low, average and high traffic demand respectively. For the computation of the number of outage users (non covered users) we defined a two steps cycle of network stabilization in which we use firstly the estimation of the RSRP and secondly the estimation of the SINR. With respect to coverage, the conclusion is that the number of users in outage in LTE is sensitive to the choice of the reuse pattern of the frequency band. The tests confirm that reuse scheme $1 \times 3 \times 3$ shows better results than reuse scheme $1 \times 3 \times 1$. These tests allow us to validate our interference model and show the huge impact of frequency pattern on the capacity of the network due to resource radio limitations.

As perspectives, we aim in the remaining of this study at analyzing the influence of the load factor throughout the capacity performance metric and show the overloaded cells which represent the bottlenecks of the network. We will try also to study the impact of some antenna parameters like mechanical, electrical tilts and transmission power toward the interference model and different performance metrics (throughput, capacity, and coverage). Furthermore, robust optimization approach will be studied to highlight the impact of the traffic uncertainty in the deployment of the network.

\section{REFERENCES}

[1] X. Mao, A. Maaref and K.Teo, "Adaptive soft frequency reuse for inter-cell interference coordination in SC-FDMA based 3GPP LTE uplinks," Proc. IEEE Global Telecommunications Conference (GlobeCom), New Orleans LO, USA, Dec 2008.

[2] M. Rahman and H. Yanikomeroglu, "Enhancing cell edge performance: A downlink dynamic interference avoidance scheme with inter-cell coordination," IEEE Transaction on Wireless Telecommunication, vol.9, no.4, pp. 1414-1425, April 2010.

[3] M. Rahman and H. Yanikomeroglu, "Interference Avoidance With Dynamic Inter-Cell Coordination for Downlink LTE System," Proc. IEEE Wireless Communication and Networking Conference (WCNC), April 2009.

[4] 3GPP R1-050738, "Interference mitigation- Considerations and results on frequency reuse" Siemens, September 2005

[5] 3GPP R1-050507, "Soft Frequency Reuse Scheme for UTRAN LTE," Huawei, May 2005.

[6] O.N.C. Yilmaz, S. Hamalainen and J. Hamalainen, "System level analysis of vertical sectorisation for 3GPP LTE." Proc. IEEE 6th International Symposium On Wireless Ccommunication System (CSWCS ). September 2009.

[7] I. Siomina, P. Varbrand and D. Yuan, "Automated optimization of service coverage and base station antenna configuration in UMTS networks." IEEE Wireless Communications. Vol 13. no. 6. pp. 16-25, December 2006.

[8] R. Schoenen, W. Zirwas and B.H Walke., "Capacity and coverage analysis of a 3 GPP-LTE multihop deployment scenario," Proc.
IEEE International Conference On Communications Workshops, May 2008.

[9] H. Holma and A. Toskala, LTE for UMTS OFDMA and SCFDMA Based Radio Access, John Wiley \& sons Ltd Edition. August 2009.

[10] O.N.C. Yilmaz and S. Hamalainen, "Comparaison of remote electrical and mechanical antenna downtilt performance for $3 \mathrm{GPP}$ LTE," Proc. IEEE 70th Vehicular Conference Fall (VTC-2009 FALL), September 2009.

[11] A. Gondran, O. Baala, A. Caminada, H. Mabed, «Interference management in IEEE 802.11 frequency assignment", Proc. IEEE Vehicular Technology Conference (VTC Spring), pp. 2238-2242, Singapore, May 2008.

[12] Mabed, H., Caminada, A., "Geometric criteria to improve the interference performances of cellular network", Proc. 64th IEEE Vehicular Technology Conference (VTC Fall), pp. 1-5, Montreal, Sept. 2006.

[13] Altman, Z., Picard, J.M., Ben Jamaa, S., Fourestie, B., Caminada, A., Dony, T., Morlier, J.F., Mourniac, S., "New challenges in automatic cell planning of UMTS networks", Proc. 56th IEEE Vehicular Technology Conference (VTC Fall), pp. 951-954, Dec. 2002 . 